\title{
INVASÃO LINFÁTICA CLINICAMENTE NÃO DETECTÁVEL DO CÂNCER VULVAR
}

luis Otávio Zanatta Sarian*, Priscila Silva Marshall, Sophie françoise Mauricette Derchain, José Carlos Campos Torres, Adriana de Cassia Paiva Santos, Gustavo Antônio de Souza

Departamento de Tocoginecologia da Faculdade de Ciências Médicas da Universidade Estadual de Campinas (UNICAMP), Campinas, SP.

\footnotetext{
*Correspondência:

Rua Alexandre Fleming,

848, Nova Campinas,

Campinas, São Paulo,

Cep: $\quad 13092-340$.

sarian@fcm.unicamp.br
}

\begin{abstract}
RESUMO
OBjetivo. Avaliar em mulheres com carcinoma escamoso da vulva menor que $5 \mathrm{~cm}$ e clinicamente sem comprometimento inguinal a invasão por neoplasia nos linfonodos inguinais superficiais e profundos.

Mitrodos. Foram avaliados os dados de 59 mulheres atendidas entre outubro de 1982 e janeiro de 2004 na Universidade Estadual de Campinas, em decorrência de carcinoma escamoso invasivo da vulva T1 ou T2 e com linfonodos inguinais clinicamente livres de invasão neoplásica (N O). Foram levantadas características clínicas do tumor e das pacientes e os dados do seguimento. Foram calculados os odds ratio e teste exato de Fisher para as associações entre a invasão dos linfonodos inguinais com o tamanho do tumor, grau histológico, recidivas e complicações. A confiança estatística foi de $95 \%$.

Resultados. A idade das mulheres variou de 34 a 91 anos (média de 67 anos), com tempo de seguimento entre três dias (óbito perioperatório) e 252 meses (média de 27 meses). Clinicamente, 22 (37\%) mulheres apresentavam tumores T1 e 37 (63\%) T2. Após análise histológica, seis (10\%) mulheres apresentavam invasão unilateral e três (5\%) bilateral, não havendo associação entre o tamanho do tumor e a invasão dos linfáticos inguinais. Também o tamanho do tumor à avaliação patológica e seu grau histológico não se mostraram associados à invasão nos linfonodos inguinais. Recidivas e complicações tardias não se correlacionaram com a invasão neoplásica inguinal.

Conclusōes. A dissecção inguinal superficial e profunda revelou invasão neoplásica clinicamente não detectável em $15 \%$ das mulheres estudadas, apesar de que tamanho e grau histológico do tumor, recidivas e complicações tardias não estiveram associadas com a invasão nos linfonodos.
\end{abstract}

Unitermos: Câncer da vulva. Linfonodos. Metástases. Cirurgia.

\section{INTRODUÇÃO}

0 câncer de vulva corresponde a menos de $1 \%$ das neoplasias malignas da mulher e a $3 \%$ a $5 \%$ dos tumores genitais femininos ${ }^{1,2}$. Poucos são os serviços capazes de compilar número substancial de casos, to rnando o estudo de seu tratamento e evolução tarefa difícil. Também, a pequena casuística faz com que a capacitação de pessoal para lidar com esta neoplasia seja exígua e restrita a centros de oncologia ginecológica com grande porte.

A forma invaso ra da doença costuma ocorrer em mulheres após a menopausa, com idade acima de 50 anos, sendo que $50 \%$ se apresentam com 70 anos ou mais no momento do diagnóstico ${ }^{3}$. Entretanto, essa neoplasia pode acometer pacientes mais jovens, sendo descritas séries com $12 \%$ a $15 \%$ dos casos antes dos 45 anos 4 . A disseminação da neoplasia invasora pode ocorrer nos tecidos adjacentes ao tumor primário, por embolização para linfonodos regionais, usualmente inguinais superficiais e profundos e eventualmente pélvicos; e mais raramente pela via hematológica, atingindo pulmões, fígado e $0550 \mathrm{~S}^{1,5}$.

0 tratamento do câncer da vulva é essencialmente cirúrgico. Segundo D isaia e Creasman (1989)4, no início do século as mulheres eram submetidas apenas à ressecção local. Em 1912, Basset propôs a remoção em bloco da vulva e regiões inguinais. Taussing (1940) e Way (1948) foram responsáveis pela divulgação das técnicas cirúrgicas em monobloco, com retirada da vulva com amplas margens cirúrgicas além do tumor e das regiões inguinais, utilizando incisão única.

Hoje, alguns autores contestam anecessidade da ressecção de toda a vulva e regiões inguinais ${ }^{1,4,6-13}$. Ainda que a taxa de sobrevida das mulheres com linfonodos inguinais negativos seja muito boa, pacientes que desenvolvem recidivas em linfonodos não removidos na cirurgia inicial apresentam alta mortalidade ${ }^{12}$. Assim, outros autores tentam descrever técnicas menos agressivas para a realização da dissecção linfonodal: Micheletti et al. (2002) ${ }^{6}$ recomendaram não estender a ressecção lateral além da espinha ilíaca anterior superior; Rouzier et al. $(2002)^{8}$ sugeriram a preservação dafáscialata e veia safenae Hullu e Van Der Zee $(2003)^{13}$ advogam pela retirada e congelação do linfonodo sentinela como técnica promissora de estadiamento.

Para melhor estabelecer a segurança das técnicas de preservação linfática inguinal superficial e profunda, é relevante conhecer a prevalência de invasão neoplásica na região inguinal em mulheres com linfonodos clinicamente sem doença. Dessa forma, o objetivo do presente estudo foi ivaliar o comprometimento de linfonodo singuinais superficiais e profundos em mulheres com carcinoma escamoso da vulva menor que $5 \mathrm{~cm}$ - T1 ou T2, segundo a classificação da Federação Internacional de Ginecologia e 0 bstetrícia (FIGO ) - e com linfonodos inguinais não palpáveis ou clinicamente livres de invasão neoplásica, submetidas inicialmente a tratamento cirúrgico com dissecção linfática inguino-femoral superficial e profunda bilateral. 


\section{METOdOS}

\section{Seleção das mulheres}

Para este estudo clínico foram avaliados os prontuários de 59 mulheres atendidas no Centro de Atenção Integral à Saúde da Mulher da Universidade Estadual de Campinas (CAISM-UN ICAMP), em decorrência de carcinoma escamoso invasivo da vulva, com tumores clinicamente menores que $2 \mathrm{~cm}(T 1)$ ou entre $2 \mathrm{~cm}$ e $5 \mathrm{~cm}$ (T2) e com linfonodos inguinais não palpáveis ou clinicamente livres de invasão neoplásica (N O). Para avaliar as recidivas nestas mulheres, foram incluídas pacientes operadas entre outubro de 1982 e setembro de 2001, sendo que o período de seguimento analisado foi da data da primeira cirurgia até janeiro de 2004. 0 s dados dos prontuários foram transferidos para fichas codificadas e posteriormente tabulados em planilhas eletrônicas (Excell囚).

\section{Variáveis estudadas}

Foram levantados os seguintes dados referentes às pacientes: idade, tamanho clínico e histológico iniciais, classificados segundo 0 estadiamento proposto pela FIGO $(1996)^{14}$, tipo de cirurgia realizada (vulvectomia radical, em monobloco ou com três incisões, ou vulvectomia parcial com esvaziamento dos linfonodos inguinais superficiais e profundos bilaterais), data da cirurgia, avaliação do grau histológico do tumor ${ }^{15}$ e presença de invasão histológica dos linfonodos inguinais. Também foram avaliados 0 aparecimento de complicações tardias decorrentes do tratamento (linfedema e retração cicatricial) e a ocorrência de recidivas da doença. Foram levantadas a data mais recente de comparecimento à consulta ambulatorial ou a data do óbito. 0 tempo de seguimento foi calculado com base na diferença entre a data da cirurgia e a da última consulta ou óbito.

\section{Análise estatística}

Para a avaliação da relação entre o tamanho do tumor ao exame clínico e a invasão dos linfonodos inguinais, foi aplicado o teste exato de Fisher. Foram calculados os odds ratio para as associações entre a invasão dos linfonodos inguinais e as seguintes características: tamanho do tumor no exame histológico, grau histológico, ocorrência de recidivas e de complicações tardias. Todos os cálculos foram realizados com intervalos de confiança estatística de 95\% (IC 95\%), através do programa Epilnfo v.3.0 para microcomputadores (Centers for Disease Control, Atlanta, Estados Unidos).

\section{RESULTADOS}

A idade das mulheres, no momento da cirurgia, variou de 34 a 91 anos (média de 67 anos). Trinta e nove (66\%) mulheres foram tratadas com vulvectomia radical em três incisões, $17(29 \%)$ com vulvectomia com incisão em monobloco e três (5\%) com vulvectomia parcial/tumorectomia e esvaziamento linfático inguinal superficial e profundo bilateral. 0 tempo médio de seguimento variou de três dias (óbito perioperatório) a 252 meses, com média de 27 meses (dados não mostrados em tabelas).

Clinicamente, 22 mulheres (37\%) apresentavam tumores menores que $2 \mathrm{~cm}$ (T1) e 37 (63\%) tumores entre $2 \mathrm{~cm} \mathrm{e} 5 \mathrm{~cm}$ (T2). Entre as mulheres com tumores $T 1,19$ (86\%) estavam livres de invasão dos linfonodos inguinais, enquanto duas ( $9 \%$ ) apresentaram invasão unilateral e uma ( $5 \%$ ) invasão bilateral. Entre as mulheres com tumores T2, $31(83 \%)$ não apresentavam invasão por neoplasia nos linfonodos inguinais, porém quatro (11\%) tinham comprometimento unilateral e duas $(6 \%)$ bilateral. N ão houve associação significativa entre o tamanho do tumor e a invasão dos linfáticos inguinais (Tabela 1).

Ao considerarmos as dimensões do tumor a partir da avaliação histológica (Tp), 21 (36\%) tinham tumores menores que $2 \mathrm{~cm}$ (Tp1) e 38 (64\%) tumores de $2 \mathrm{~cm} \mathrm{a} 5 \mathrm{~cm}$ (Tp2). Das mulheres com Tp1, 14\% apresentavam comprometimento inguinal, enquanto 15\% das com Tp2 também apresentavam esta condição. A maioria $(69 \%)$ apresentou tumores bem diferenciados (GI), e apenas três mulheres (5\%) tinham tumores pouco diferenciados. Não houve associação significativa entre o tamanho do tumor (Tp) ou seu grau de diferenciação e o comprometimento dos linfonodos inguinais (Tabela 2).

Treze mulheres $(22 \%)$ apresentaram recidivas durante 0 seguimento. Dessas, uma (8\%) ocorreu na região inguinal - no local da dissecção linfática - embora esta paciente não apresentasse invasão dos linfonodos. Duas mulheres (16\%) tiveram recidivas fora da região operada (no pulmão e fígado) e 10 (76\%) desenvolveram recidivas vulvares, sendo que duas dessas mulheres possuíam extensão da doença para a região inguinal. D ezesseis $(27 \%)$ apresentaram complicações tardias secundárias ao tratamento (linfedema) e duas pacientes tiveram retração da cicatriz inguinal. A evolução da doença (recidivas) e as repercussões de sua evolução e tratamento (complicações tardias) não estiveram significativamente associadas com a invasão dos linfonodos inguinais (Tabela 3).

\section{DISCUSSÃo}

No presente estudo, cerca de $20 \%$ das 59 mulheres avaliadas apresentaram comprometimento neoplásico dos linfonodos inguinais superficiais ou profundos, apesar de não manifestarem anormalidades da inspeção e palpação de suas regiões inguinais. Ainda mais relevante, um terço das mulheres com disseminação inguinal superficial ou profunda histologicamente confirmada tinha comprometimento nodal bilateral (N 2).

Dados compilados de seis instituições, totalizando 451 mulheres com carcinoma escamoso da vulva e que foram submetidas à dissecção linfática inguinal superficial ou profunda, mostraram que 19,5\% das mulheres com região inguinal clinicamente normal tiveram linfonodos com invasão histológica, contrastando com proporção semelhante $(21,8 \%)$ de mulheres com suspeita clínica de invasão e que tinham os linfonodos inguinais histologicamente livres da doença ${ }^{16}$. Vale ressaltar que estes dados incluíram mulheres operadas entre 1940 e 1986 e em diferentes serviços, o que pode conferir à amostra grande variabilidade técnica em termos de estratégia operatória e processamento e avaliação histológica. Ainda assim, a proporção de mulheres clinicamente negativas para disseminação linfática inguinal superficial ou profunda é muito semelhante comparando nossos resultados com o dessa compilação.

A disseminação linfática do carcinoma escamoso da vulva parece estar relacionada ao tamanho do tumor no momento do diagnóstico, 


\begin{tabular}{|c|c|c|c|}
\hline \multirow[b]{2}{*}{ Tamanho clínico do tumor $(\mathrm{T})$} & Avaliaçāo histológica dos & \multicolumn{2}{|c|}{ linfonodos inguinais superficiais e profundos } \\
\hline & Sem invasão $(\mathrm{NpO})$ & Com invasão unilateral (Np1) & Com invasão bilateral (Np2) \\
\hline $\begin{array}{l}<2 \mathrm{~cm}(\mathrm{~T} 1) \\
2 \text { a } 5 \mathrm{~cm}(\mathrm{~T} 2) \\
\text { Total }\end{array}$ & $\begin{array}{c}N(\%) \\
19(38) \\
31(62) \\
50(100)\end{array}$ & $\begin{array}{l}N(\%) \\
2(33) \\
4(67) \\
6(100)\end{array}$ & $\begin{array}{l}N(\%) \\
1(33) \\
2(67) \\
3(100)\end{array}$ \\
\hline
\end{tabular}

Tabela 2 - Características anatomopatológicas do tumor e invasäo dos linfonodos inguinais

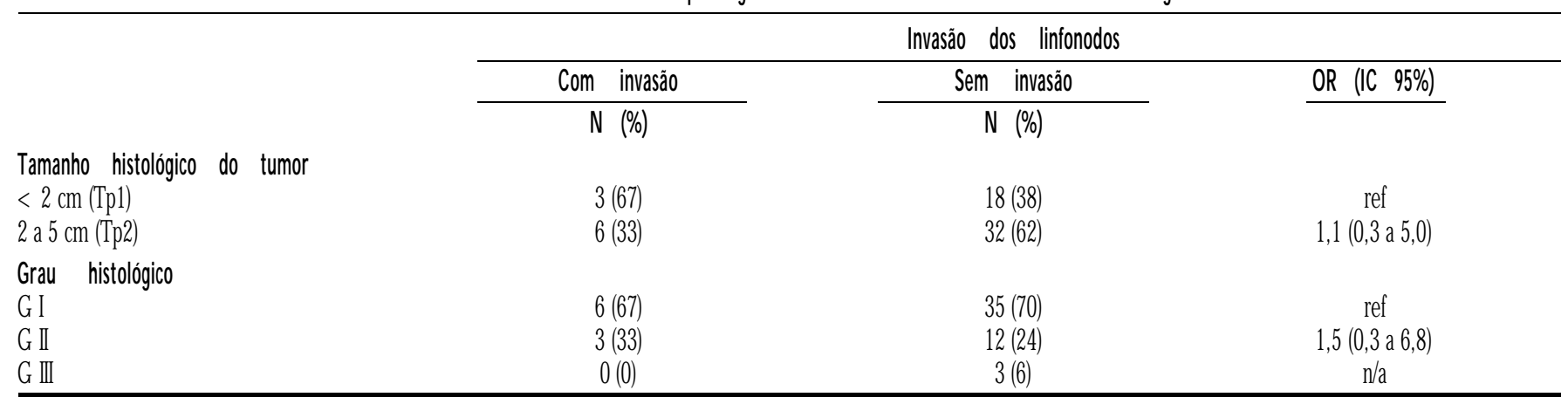

\begin{tabular}{|c|c|c|c|}
\hline & \multicolumn{3}{|c|}{ Linfonodos inguinais } \\
\hline & Com invasão & Sem invasão & OR (IC 95\%) \\
\hline & $\mathrm{N}(\%)$ & $\mathrm{N} \quad(\%)$ & \\
\hline 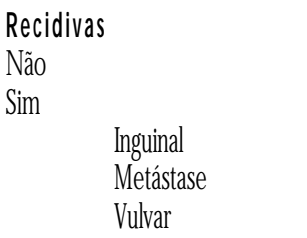 & $\begin{array}{l}6(67) \\
3(33) \\
0(0) \\
1(50) \\
2(20)\end{array}$ & $\begin{array}{l}40(80) \\
10(20) \\
1(100) \\
1(50) \\
8(80)\end{array}$ & $\begin{array}{c}\text { ref } \\
0,5(0,1 \text { a 2,3) }\end{array}$ \\
\hline $\begin{array}{l}\text { Complicaçōes tardias } \\
N \text { ão } \\
\text { Sim } \\
\quad \text { Linfedema } \\
\text { Retração cicatricial }\end{array}$ & $\begin{array}{l}5(56) \\
4(44) \\
4(25) \\
1(50)\end{array}$ & $\begin{array}{l}38(76) \\
12(24) \\
12(75) \\
1(50)\end{array}$ & $\begin{array}{c}\text { ref } \\
0,4(0,1 \text { a } 1,7)\end{array}$ \\
\hline
\end{tabular}

estando invadidos pela neo plasia os linfono dos inguinais superficiais ou profundos em cerca de $10 \%$ das mulheres com tumores menores que $2 \mathrm{~cm}$ e em aproximadamente $26 \%$ daquelas com tumores maiores que $2 \mathrm{~cm}$ e restritos à vulva ${ }^{1,3,5,7,8,17}$. Em nosso estudo, as proporções de invasão nodal foram estatisticamente semelhantes nos grupos de mulheres com tumores T1 ou T2. Também, quando a avaliação do tamanho tumoral foi realizadanos estudos histológicos (Tp), não houve diferenças significativas entre as proporções de linfonodos inguinais invadidos comparando o grupo Tp1 com Tp2. Deve-se salientar, contudo, que 0 número de mulheres da casuística provavelmente contribuiu para essa ausência de diferencial em relação ao tamanho do tumor, caracterizando um dos maio res problemas no estudo do câncer de vulva: 0 pequeno número de casos para compilação.

Com relação ao tumor, ao lado do tamanho, o grau de diferenciação histológica também não encontrou correlação significativa com a prevalência de comprometimento linfonodal inguinal. Ao abordar este aspecto, vale recordar que os tumores escamosos vulvares se subdividem em do is grupos cujas etiogenias são provavelmente diferentes: uma porção das lesõesacomete mulheresmais idosas (com idade entre 55 e 85 anos), sendo estes tumores altamente queratinizados e 
etiopatologicamente associados com líquen escleroso, hiperplasia escamosa e neoplasia intra-epitelial vulvar bem diferenciada (N IV1), sem relação com o Papilomavírus humano (HPV); a segunda porção acomete mulheres mais jovens (com idade entre 35-65 anos), sendo de características basalóides e associada à neoplasia intra-epitelial vulvar de alto grau (N IV3), provavelmente decorrente da infecção pelo HPV ${ }^{18-21}$. Em nosso estudo, embora a média etária das mulheres tenha sido relativamente elevada (67 anos de idade), aproximadamente um quarto delas tinha 55 anos de idade ou menos. Assim, temos em nossa amostragem dois grupos de lesões com etiopatogenia diferenciada, com predomínio dos tumores bem queratinizados e diferenciados - mais de $60 \%$ dos casos incluídos em nosso estudo foi de tumores $G$ I. Dessa forma, apenas ampliando substancialmente o número de mulheres jovens seria possível obter proporções equivalentes de tumores bem e pouco diferenciados, 0 que eventualmente poderia revelar diferenças significativas de comprometimento dos linfonodos inguinais em função do grau de diferenciação histológica.

As mulheres em nosso estudo foram submetidas a linfadenecto mia inguinal superficial e profunda. No os últimos 30 anos, existe tendência cada vez mais acentuada entre pesquisadores e cirurgiões em reduzir a extensão do tratamento cirúrgico, sobretudo procurando reduzir a morbi-mortalidade associada à dissecção inguinal22,23. 0 grande impulso para 0 tratamento conservador das regiões inguinais ocorreu quando D iSaia et al. (1979) ${ }^{24}$ propuseram a excisão local radical e dissecção inguinal superficial, para pacientes selecionadas. D esde então, tornando-se comum a prática da conservação de linfáticos inguinais profundos, sobretudo em mulheres com tumores T1, grande experiência foi adquirida em relação à disseminação da doença para esta região. Ao analisar dados de 104 pacientes com tumores T1 e T2 operadas entre 1986 e 1997 e que foram submetidas a dissecção inguinal superficial, Gordinier et al. $(2003)^{25}$ encontraram cerca de $9 \%$ de recidivas inguinais, em média 22 meses após o tratamento inicial. Esses autores atribuem as recorrências a micrometástases presentes na região inguinal profunda, concluindo que a dissecção superficial da região inguinal superficial e profunda não previne adequadamente recorrências não locais. Vale ainda dizer que 0 prognóstico para mulheres com recidivas inguinais é ruim, evoluindo para óbito na maior parte dos $\operatorname{casos}^{23}$.

A maior parte das recidivas encontradas na presente casuística ocorreu na região vulvar. A freqüência de recorrências da doença varia de $12 \%$ a $37 \%$ das mulheres, sendo que os fatores com maior poder de predição das recidivas são o tamanho da lesão, margens excisionais exíguas $(<1 \mathrm{~cm})$ e 0 comprometimento linfonoda 80,26 . Também relevante é o fato de que as recorrências vulvares e perineais foram mais freqüentes, nesta compilação, entre mulheres com linfonodos inguinais positivos, o que não se reproduziu em nossa casuística. As proporções para o local de recorrência foram $53 \%$ isoladamente na vulva/períneo, $19 \%$ inguinais, $8 \%$ à distância e $18 \%$ em múltiplos locais concomitantemente. Essas proporções se assemelham às por nós encontradas, à exceção dos casos com múltiplos locais de recorrência, que não ocorreram no grupo de mulheres que estudamos.

\section{CONCLUSÕES}

A abordagem conservadora da região inguinal superficial e profundaaindaé objeto de estudo. N ossos dados, em paralelo aosde estudos prévios, sugerem que o cirurgião não pode se fiar no exame clínico da região inguinal para determinar sua conduta. A justificada preocupação em resguardar a paciente das complicações decorrentes da dissecção completa dos linfonodos inguinais superficial e profunda deve levar em consideração a chance de ocorrerem recidivas, em geral fatais, nos linfonodos não dissecados. U ma vez que a simples dissecção linfática superficial não se mostrou efetiva em outros estudos, como previamente discutido neste artigo, ganha relevância a técnica do linfonodo sentinela, ainda em estudos, que pode prover uma abordagem conservadora e segura ${ }^{11,27,28}$. A associação de cirurgia e radioterapia também mostra resultados alentadores $5^{9,10}$ mas, enquanto a detecção dos carcinomas de vulva não se restringir a casos iniciais, provavelmente mutilações e complicações sérias continuarão a acometer as pacientes.

Conflito de interesse: não há.

\section{SUMMARY}

\section{Clinically undetectable LYMph node invasion IN vulvar} CANCER

$O$ BJECTIVE. TO assess the neoplastic invasion of superficial and deep inguinal lymph nodes of women with invasive vulvar squamous carcinoma smaller than 5 centimeters with a clinically normal inguinal region. Methods: the medical records of 59 women cared at the State U niversity ofC ampinas with invasive vulvar squamous carcinoma $\mathrm{T} 1$ and $\mathrm{T} 2$ and who presented clinicallynormal inguinal regions (N 0 ) were reviewed. Clinical characteristics of both tumor and patients were evaluated as well as the follow-up data. 0 dds ratiosand Fisher's Exact Test w ere used to assess the correlations between the invasion of inguinal lymph nodesand tumor size, grade, relapsesand clinical complications. Confidence limits of $95 \%$ were used.

RESULtS. Age of the patients ranged from 34 to 91 years (mean 67 years), and follow- up time ranged from 3 days (peri-operatory death) to 252 months (mean 27 months). Clinically, 22 (37\%) women had lesions T1 lesionsand 37 (63\%) ) T2. H istological analysis showed unilateral lymphatic invasion in six (10\%) women and bilateral in three (5\%). There was no significant association between tumor size and lymph node invasion. Also, pathologic tumor size and grade were not asso ciated with lymph nodeneoplastic involvement. Relapsesand late complications were notcorrelated with lymph nodeneoplastic invasion.

Conclusions. Superficial and deep inguinal dissection disclosed clinically undetectable lymph node neoplastic invasion, although tumor size and histological grade, relapses and late complications were not associated with node involvement. [Rev Assoc Med Bras 2005; 51(4): 228-32]

KEY WORDS: Vulvar cancer. Lymph nodes. Metastasis. Surgery.

\section{REFERÊNCIAS}

1. Coleman RL, Santoso JT. Vulvar carcinoma. Curr Treat O pion O ncol 2000;1:177-90. 
2. Verdiani LA, Juliato CR, D erchain SFM . C arcinoma da vulva: epidemiologia e aspectos clínicos. Rev Centro de Referência 1997;2:86-90.

3. Canavan TP, Cohen D. Vulvar cancer. Am Fam Physician 2002;66:126974

4. Disaia PJ, Creasman WT. Invasive cancer of the vulva. In: Disaia PJ, Creasman WT. Clinical gynecologic oncology. $3^{\text {rd }}$ ed. St Louis: Mosby; 1989. p.241-72.

5. De Hullu JA, Hollema H, LolkemaS, Boezen M, Boonstra H, Burger MO, et al. Vulvarcarcinoma: the price of less radical surgery. Cancer 2002;95:2331-8.

6. Micheletti L, Levi AC, Bogliatto F, Preti M, Massobrio M. Rationale and definition of the lateral extension of the inguinal lymphadenectomy for vulvar cancer derived from an embriological and anatomical study. J Surg Oncol 2002;81:19-24.

7. Kim RY, Alvarez RD, O mura GA. Advances in the treatment of gynecologic malignancies. Part 1: cancers of the cervix and vulva. Oncology 2002;16:1510-7.

8. Rouzier R, Haddad B, Plantier F, Dubo is P, Pelisse M, Paniel BJ. Local relapse in patients treated for squamous cell vulvar carcinoma: incidence and prognostic value. 0 bstet Gynecol 2002;100:1159-67.

9. Edwards S, Handfield-Jones S, Gull S. National guidelines on the management of vulval conditions. Int J STD AIDS 2002;13:411-5.

10. U litin HC, Pak Y, D ede M. Can radiotherapy be a treatment option for elderly w omen w ith invasive vulvar carcino ma without radical surgery? Eur J Gynaecol O ncol 2002;23:426-8.

11. Puig-Tintore LM, O rdi J, Vidal-Sicart S, Lejarcegui JÁ, Torn A, Pahisa J, et al. Further data on the usefulness of sentinel lymph node identification and ultrastaging in vulvar squamous cell carcinoma. Gynecol O ncol 2003:88:29-34

12. Scheiströen $M, N$ esland JM, Trope $C$. H ave patients with early squamous carcinoma of the vulva been overtreated in the past? The norvegian experience 1977-1991. Eur J Gynaecol O ncol 2002;23:93-103.

13. H ullu JA, Van D er Zee AG. Sentinel node techniques in cancer of the vulva. Curr W omens Health Rep 2003;3:19-26.

14. Shepherd JH. Cervical and vulvar cancer: changes in FIG O definitions of staging. Br J O bstet Gynecol 1996;103:405-6.

15. Scully RE, Bonfligio TA, Kurman RJ, Silverberg SG, W ilkins EJ. H istological typing of female genital tract tumors. In: World Health O rganization. International. $\mathrm{H}$ istological classification of tumors. 2th ed. Berlin: SpringerVerlag; 1994
16. Bosquet JG, Kinney W K, Russel AH, G affey TA, Magrina JF, Podratz KC Risk of occult inguinofemoral lymph node metastasis from squamous carcinoma of the vulva. Int J Radiat O ncol Biol Phys 2003;2:419-24.

17. Gomes-Rueda N, Vighi S, Garcia A, C ardinal L, Belardi M G, Di Paola G $\mathrm{H}$ istologic predictive factors: therapeutic impact in vulvar cancer. J Reprod Med 1994;39:71-6.

18. Crum CP. Carcinoma of the vulva: epidemiology and pathogenesis. O bstet Gynecol 1992;79:448-54.

19. Kurman RJ, Trimble $C L$, Shah KV. Human papilloma virus and the pathogenesis of vulvar carcinoma. Curr O pin Obstet Gynecol 1992:4:582-5

20. Trimble $C L$, Hildenshein $A$, Brinton $L A$, Shah $K V$, Kurman RJ. Heterogenous etiology of squamous carcinoma of the vulva. $O$ bstet Gynecol 1996;87:59-64.

21. AlG hamdi A, Freedman D, Miller D, Poh C, Rosin M, Zhang L, et al. Vulvar squamous cell carcinoma in young women: a clinico pathologic study of 21 cases. Gynecol O ncol 2002; 84:94-101.

22. Morris JM. A formula for selective lymphadenectomy: its application to cancer of the vulva. O bstet $\mathrm{G}$ ynecol 1977;50:152-8.

23. Hacker N F. Management of regional lymph nodes and their prognostic influence in vulvar cancer. O bstet G ynecol 1983;61:408-12.

24. DiSaia PJ, C reasman WT, Rich W M. An alternate approach to early cancer of the vulva. Am J O bstet Gynecol 1979;133:825-32.

25. Gordinier ME, Malpica A, Burke TW, Bodurka D C, W olfJK, Jhingran A, et al. Gro in recurrence in patients with vulvar cancer with negative nodes on superficial inguinal lymphadenectomy. G ynecol O ncol 2003;90:625-8.

26. Tilmans AS, Sutton GP, Look KY, Sethman FB, Ehrilich CE, H ornback N B. Recurrent squamous carcinoma of the vulva. Am J O bstet Gynecol 1992;167:1383-9.

27. Levenback C, Burke TW, Gershenson D M, Morris M, Malpica A, Ross MI. Intraoperative lymphatic mapping for vulvar cancer. O bstet Gynecol 1994;84:163-7.

28. Terada KY, Shimizu DM, Wong JH. Sentinel node dissection and ultrastaginc in squamous cell cancer of the vulva. Gynecol 0 ncol 2000;76:40-4

Artigo recebido: 22/04/04 Aceito para publicação: 28/07/04 